\title{
Travellers' diarrhoea
}

\author{
Mostly due to bacteria and difficult to prevent
}

The days are past when travellers' diarrhoea can be attributed to over indulgence in local food or wine, "a change in the water," or merely "travellers' nerves" relating to the trials and tribulations of international travel. At least $70 \%$ of episodes of diarrhoea occurring in travellers leaving their homeland, usually the industrialised West, for less well developed parts of the world are due to an infective agent.

$\mathrm{Up}$ to one half of these episodes are due to enterotoxin producing Escherischia coli, the remainder being due to other bacterial enteropathogens such as Shigella spp, Salmonella spp and other varieties of $E$ coli (such as enterohaemorrhagic $E$ coli and enteroinvasive $E$ coll); viruses including the Norwalk family of viruses; and intestinal parasites such as Giardia lamblia, Cryptosporidium parvum, and Entamoeba histolytica. ${ }^{1}$ New enteropathogens continue to emerge. Microsporidium sp (Enterocytozoon bieneusi) is now recognised as an important cause of diarrhoea in people infected with HIV and will probably surface as an uncommon cause of diarrhoea in immunocompetent travellers. A new coccidian parasite, which resembles cyanobacteria in appearance and may eventually assume the name of Cyclospora cayetanensis, was first described in travellers in Nepal and has now been identified in small intestinal epithelial cells of two travellers with diarrhoea. ${ }^{2}{ }^{3}$

These infections are most commonly acquired through ingestion of contaminated food and water but may be transmitted by person to person contact. Swimming pools are occasionally responsible for transmission, particularly of $C$ parvum and $G$ lamblia, which can even survive in chlorinated water for limited periods. Despite the traditional "healing properties" of seawater, many beaches around the world are now contaminated with sewage and faecal micro-organisms. ${ }^{4}$ A recent survey of Ramsgate beach in Kent confirmed that diarrhoea and other abdominal symptoms were more common in bathers than non-bathers and that the coliform count failed to achieve European Community standards on $12 \%$ of the sampling occasions. ${ }^{5}$

Inland lakes and rivers are generally not approved for recreational swimming and are not routinely monitored. Recent evidence confirms that more than $80 \%$ of freshwater locations that had been tested in Britain were contaminated with cyanobacterial toxins, for which the increasing use of nitrate and phosphate fertilisers and a series of long, hot summers are thought to account. ${ }^{4}$ Holiday makers are advised to refer to the Heinz Good Beach Guide for up to date information on seawater contamination. ${ }^{6}$
Most episodes of travellers' diarrhoea are self limiting and do not require any specific treatment. In most cases the illness can be allowed to run its course, with symptomatic treatment alone and no need to isolate the pathogen. ${ }^{17}$ Exceptions are bloody diarrhoea (dysentery) and diarrhoea lasting longer than seven days. Antibiotics are indicated for amoebic dysentery and in severe bacterial dysenteries with systemic illness. Similarly, travellers with persistent diarrhoea, possibly due to giardiasis or tropical sprue, would benefit from antimicrobial chemotherapy.

For the watery diarrhoeas, the cornerstone of treatment is replacing lost fluid and electrolytes either by formal oral rehydration therapy with glucose-electrolyte solutions (mandatory for infants and young children and possibly for elderly patients) or informally by encouraging a high fluid intake, which should include salty soups (sodium), fruit juices or bananas (potassium), and a source of complex carbohydrates (bread, rice, potato, or salty crackers) to promote active glucose-sodium cotransport.

Antidiarrhoeal preparations such as loperamide (preferred to natural opiates as it has almost no effect on the central nervous system) can reduce stool frequency and improve stool consistency. Although loperamide has some antisecretory activities in vitro, its clinical benefits in humans depend largely on its inhibitory effect on small intestinal motility. Loperamide should not be used in infants and young children because of the rare but real risk of respiratory depression and because it may detract from the life saving intervention of oral rehydration therapy. Antidiarrhoeal drugs should be avoided in dysentery because of concerns about colonic dilatation. Recently, impressive effects in travellers' diarrhoea have been reported for zaldaride maleate, which acts by inhibiting calmodulin and thus reducing intestinal secretion of water and electrolytes. ${ }^{8}$ This drug could have an important role in symptom control in travellers' diarrhoea.

The role of antimicrobial drugs in treating travellers' diarrhoea continues to be controversial. Good evidence exists, however, that various agents, including co-trimoxazole, doxycycline, and the new 4-fluoroquinolones, including ciprofloxacin and norfloxacin given for three to five days, can reduce the duration and severity of the illness. ${ }^{17}$ These benefits must be weighed against the potential risk of adverse reactions to these drugs (including allergic reactions, photosensitivity, and antibiotic related diarrhoea) and to the cost, which for the 4-fluoroquinolones is substantial. For those of us who have experienced the dramatic relief which follows one 
or two doses of an antibiotic, however, withholding treatment that will reduce a three to five day illness to one which lasts only 12 to 24 hours is difficult. Travellers and their medical advisors should therefore develop self treatment strategies that should include advice about early oral rehydration therapy together with the self administration of loperamide and antibiotics. Medical advice, however, should be sought for severe diarrhoea and dehydration, dysentery, and persistent diarrhoea.

Prevention is always better than treatment, but sadly the standard advice for preventing travellers' diarrhoea almost always fails. Formal evaluation of Swiss travellers visiting Africa and Asia indicated that only $2 \%$ were able to consistently adhere to the strict dietary advice given. ${ }^{9}$ Even when travellers are careful they can often be caught out. Bacterial enteropathogens are able to survive in food that is too hot to touch $\left(50^{\circ} \mathrm{C}\right)^{10}$ and bacteria in ice cubes can be recovered from cocktails containing tequila, whisky, and other spirits. ${ }^{11}$

For many business travellers it is often impossible and indeed unsociable not to eat with local residents and strict control of food and beverage intake may therefore be difficult. Various antibiotics taken prophylactically will prevent travellers' diarrhoea, but most experts would not recommend this for all travellers. People at high risk of acquiring travellers' diarrhoea or with increased susceptibility to its effects might consider prophylaxis, as could the short term visitor for whom the loss of even 12 to 24 hours would seriously interfere with their visit.

Economic arguments have been produced for more liberal use of antibiotic prophylaxis, although their effects would probably militate against this. Alternative preventive measures such as bismuth preparations might also be considered, although their efficacy compares unfavourably with that of antibiotics. Work continues on vaccines to prevent travellers' diarrhoea, but as yet these are not widely available and require further evaluation before they can be recommended.

M J G FARTHING Professor of gastroenterology

Department of Gastroenterology,

St Bartholomew's Hospital,

London EC1A 7BE

1 Farthing MJG, Du Pont HL, Guandalini S, Keusch GT, Steffen R. Treatment and prevention of travellers' diarrhoea. Gastroenterology International 1992;5:162-75.

Shlim DR, Cohen MT, Taton M, Rajah R, Long EG, Ungar BL. An algae-like organism associated with an outbreak of prolonged diarrhoea among foreigners in Nepal. An f Trop Med Hyg 1991;45:383-9.

3 Bendall RP, Lucas S, Moody A, Tovey G, Chiodini PL. Diarrhoea associated with cyanobacteriumlike bodes: a new coccidian enteritis of man. Lancet 1993;341:590-2.

Walker A. Swimming - the hazards of taking a dip. $B M 7$ 1992;304:242-5.

5 Balarajan R, Raleigh VS, Yuen P, Wheeler D, Machin D, Cartwright R. Health risks associated with bathing in seawater. $B M 7$ 1991;303:1444-5.

Marine Conservation Society. Heinz good beach guide. A guide to over 500 of Britain's cleanest beaches. London: Vermilion, 1993.

7 Farthing MJG. Prevention and treatment of travellers' diarrhoea. Alimentary Pharmacology and Therapeutics 1990;5:15-30.

8 Du Pont HL, Ericsson CD, Mathewson J, Marani S, Knellwolf-Cousin A-L, Martinz-Sandoval FG. Zaldaride maleate, an intestinal calmodulin inhibitor, in the therapy of travellers' diarrhoea.

Kozicki M, Steffen R, Schar M. "Boil it, cook it, peel it or forget it": does this rule prevent travellers' diarrhoea? Int $\mathcal{F}$ Epidemiol 1985; 14:169-72.

10 Bandres JC, Mathewson JJ, Du Pont HL. Heat susceptibility of bacterial enteropathogens. Arch Intern Med 1988;148:2261-3.

1 Dickens DL, Du Pont HL, Johnson PC. Survival of bacterial enteropathogens in the ice of popular drinks. fAMA 1985;253:3141-3.

\section{Jaundice persisting beyond 14 days after birth}

\section{Requires immediate referral to a paediatrician}

This week the Children's Liver Disease Foundation launches a campaign to improve the early diagnosis of babies with liver disease. Extrahepatic biliary atresia is the commonest life threatening hepatobiliary disorder in childhood, affecting between one in 14000 and one in 21000 live born infants. 'It must be considered in any infant remaining jaundiced beyond 14 days of age $\mathrm{e}^{2}$ as the success of treatment depends on early surgery. In this condition the urine is always yellow and the stools acholic. Occasionally in infants with atresia the stools are pigmented with yellow or green pigment in the first weeks after birth before bile flow is completely obstructed. Biliary atresia is not a congenital malformation per se but an acquired progressive disease of the biliary system of unknown aetiology.

Despite many reports emphasising the need for early surgical intervention delayed referral remains the pattern for most cases. Long term survival of patients who have undergone surgical treatment early in infancy has been one of the most important advances in paediatric surgery in the past three decades.

From Thomson's first description of the condition in $1891^{3}$ until Kasai's portoenterostomy ${ }^{4}$ most cases were considered uncorrectable. Kasai's procedure, in which a Roux-en-Y loop of jejunum is anastomosed to transected, microscopic bile duct remnants in the porta hepatis, was described in 1959 but not accepted as a standard procedure in Britain until the '70s. It remains the initial choice for surgical management, providing effective bile drainage when performed in the first 2 to 3 months of life.

The development of liver transplantation has further increased the chances of curing biliary atresia. There has been debate over whether orthotopic liver transplantation or the Kasai procedure should be the first treatment tried, but most paediatric surgeons accept that Kasai's portoenterostomy is the preferred treatment. Transplantation is not jeopardised by a previous portoenterostomy and should probably be reserved for those in whom portoenterostomy fails initially or who deteriorate with progressive liver disease some months or years later. The five year survival rate of portoenterostomy is $50 \%$ to $60 \%$. The survival rate of liver transplantation in children less than 1 year of age is similar, ${ }^{5}$ although the overall survival rate in all children is over $70 \%$. It must be remembered, however, that orthotopic liver transplantation is not without attendant risks of life long immunosuppression and complications. ${ }^{6}$

All infants presenting with biliary atresia below 12 weeks of age should undergo a Kasai portoenterostomy, but beyond 12 weeks some centres immediately consider liver transplantation. The need for transplantation can also be determined by measuring the size of ductuli at the porta hepatis. ${ }^{7}$ The larger the ductuli at the porta hepatis the better the prognosis. Early recognition of the condition and early referral for surgery can reduce the number of infants requiring transplantation. Many series have shown that infants operated on before 2 months of age have a $90 \%$ chance of achieving satisfactory bile drainage. Each week after this reduces the success rate, and few cases have been successfully operated on beyond 3 months of age. The preoperative investigations to exclude other causes of jaundice take about two weeks. The following guidelines ${ }^{8}$ should be observed: 